\title{
Free-electron laser growing modes and their bandwidths
}

\author{
Stephen Webb, ${ }^{1, *}$ Vladimir N. Litvinenko, ${ }^{2,3, \dagger}$ and Gang Wang ${ }^{2, *}$ \\ ${ }^{1}$ Tech-X Corporation, 5621 Arapahoe Avenue Suite A, Boulder, Colorado 80303, USA \\ ${ }^{2}$ Collider-Accelerator Department, Brookhaven National Laboratory, Upton, New York 11973-5000, USA \\ ${ }^{3}$ Department of Physics \& Astronomy, Stony Brook University, Stony Brook, New York 11794-3800, USA
}

(Received 22 March 2012; published 7 August 2012)

\begin{abstract}
The behavior of free-electron laser amplification in the small signal linear regime can be understood by studying the dispersion relation derived from the electron energy distribution. A thorough understanding of the growth modes in this regime is of great value in understanding numerical results obtained using simulations. In this paper we show that for a typical bell-shape energy distribution in the electron beam there is not more than one growing mode. We also derive an analytical expression, which determines the bandwidth of the free-electron laser. We also discuss the limitation on the number of growing modes for the case of beam energy distributions with multiple peaks.
\end{abstract}

DOI: 10.1103/PhysRevSTAB.15.080701

PACS numbers: 41.60.Cr

\section{INTRODUCTION}

Since the invention of free-electron lasers (FELs) by Madey [1,2] the FEL field has made tremendous progress both experimentally [3-5] and theoretically. In an FEL electrons propagate through a wiggler, a device with a periodically oscillating transverse magnetic field, which causes the electrons to radiate at a resonant wavelength of

$$
\lambda_{o}=\frac{\lambda_{w}}{2 \gamma^{2}}\left(1+\left\langle a_{w}^{2}\right\rangle\right)
$$

where $\lambda_{w}$ is the wiggler period, $\gamma=E_{o} / m c^{2}$ is the relativistic factor of electrons, and $a_{w}=e A_{w} / m c$ is the normalized vector potential of the wiggler's magnetic field. A resonant interaction between the electron beam and the TEM field of the radiation can become unstable, resulting in the exponential growth of the radiation power. This results in the generation of high power pulses of coherent radiation. Such high-gain FELs are of great interest for current and future applications, and are the subject of the discussions in this paper.

Because exact analytical solutions of three-dimensional FEL equations are typically intractable, numerical codes such as GENESIS [6] and GINGER [7] are used for practical FEL designs. However, there do exist some analytical treatments [8-12] that can provide insight into the delicate phase-space dynamics of electrons, statistical characteristics of FEL radiation, and can be used for testing the validity of the assumptions made in the FEL codes. The

\footnotetext{
*swebb@txcorp.com

vl@bnl.gov

"gawang@bnl.gov
}

Published by the American Physical Society under the terms of the Creative Commons Attribution 3.0 License. Further distribution of this work must maintain attribution to the author(s) and the published article's title, journal citation, and DOI.
FEL linear regime describing the initial amplification of the density perturbation is frequently used for in-depth analysis of FEL physics, and most treatments assume a wide electron beam copropagating with the TEM wave.

Exact solutions for the growth rates of the FEL system for a cold beam and a beam with a Lorentzian energy distribution are known [10,12]. These both yield a cubic polynomial with one growing mode and two oscillating/ decaying modes.

A number of fundamental questions about FELs remain unanswered. It is understood that the self-consistent $1 \mathrm{D}$ FEL equation may have a variable number of eigenmodes ${ }^{1}$ depending upon the details of the energy distribution, with asymptotic formulas for the fastest growing mode discussed in [10-13]. A direct discussion of the diverging number of modes is given in [14].

For example, it is known that for a beam with a Gaussian energy distribution an infinite number of modes exist. But it is unknown how many of these modes are growing, or what frequency cutoffs might exist for these growing modes.

In this paper we will prove that at any given frequency a 1D FEL driven by an electron beam with a bell-shaped energy distribution (i.e. a smooth, positive, diminishing at infinity bounded function with a single maximum) has either one or no growing solutions. It means that there is only one growing eigenmode amplifying some set of frequencies, with an upper frequency cutoff. We will also derive, for the first time, an analytical expression for the amplification bandwidth of the growing eigenmode. Known roots of the cubic equations for the cases of monoenergetic and Lorentzian beams support both of these findings.

\footnotetext{
${ }^{1}$ A growing eigenmode is a solution of the self-consistent FEL equations, which grows exponentially along the length of the undulator.
} 
In Sec. II we review the FEL dispersion relation derived in the formalism developed by Saldin et al. [12]. In Sec. III we describe the main idea of the method we use to prove our findings. Section IV provides a general discussion of the technique used to analyze the results, and analyzes the case of a bell-shaped energy distribution. In Sec. V, we discuss general energy distributions with multiple peaks.

\section{1D FEL DISPERSION RELATION}

Saldin, Schneidmiller, and Yurkov provided the most general treatment of the high-gain free-electron laser operating in the small-signal, linear regime $[10,12]$. Using a Laplace transformation they reduced the self-consistent Maxwell-Vlasov equations [15] to a dispersion relation of the form

$$
s=\frac{\hat{D}(s)}{1-i \hat{\Lambda}_{p}^{2} \hat{D}(s)},
$$

where $s$ is the Laplace transformation variable along the longitudinal direction, $\hat{\Lambda}_{p}^{2}$ is a longitudinal space charge parameter, and $\hat{D}(s)$ is the dispersion integral given by

$$
\hat{D}(s)=\int d \hat{P} \frac{d \hat{F}}{d \hat{P}} \frac{1}{s+i(\hat{P}-\hat{\Delta})},
$$

where we use the conventions in [12] of $\hat{P}=\left(E-E_{o}\right) /$ $\left(\rho E_{o}\right)$ as the normalized energy deviation from the central energy of electrons, $\hat{\Delta}=L_{G}\left[\omega\left(1+a_{w}^{2}\right) /\left(2 c \gamma^{2}\right)-k_{w}\right]$ the normalized detuning from the FEL resonance ${ }^{2}(1)$, and $\hat{F}$ the normalized energy distribution of electrons. We use for normalization the e-fold FEL gain length, $L_{G}$, and the Pierce parameter, $\rho=\left(k_{w} L_{G}\right)^{-1}$, where $k_{w}=2 \pi / \lambda_{w}$ is the wiggler wave number.

The authors also found analytical solutions for the case of the monoenergetic and Lorentzian energy distributions as roots of a cubic equation and expressed the solution for the system in the form

$$
V(z)=\sum_{n=1}^{3} V_{n} e^{s_{n} z},
$$

where $z$ is the coordinate along the FEL wiggler, the $V_{n}$ contain the initial conditions, and the $s_{n}$ are the roots of the dispersion relation Eq. (2). ${ }^{3}$

In the case of an arbitrary energy distribution, there can be a finite or infinite number of roots of Eq. (2). In the general case of $N$ roots, the self-consistent FEL equation is reducible to a system of $N$ ordinary differential equations with roots of Eq. (2) being eigenvalues of the system. Naturally, the initial condition has $N$ independent

\footnotetext{
${ }^{2}$ We are using $\hat{\Delta}$ to describe the normalized detuning from FEL resonance. It has the same absolute value, but the opposite sign from convention used in $[10,12]$, i.e. $\hat{\Delta} \equiv-\hat{C}$. We think that this sign is natural, since higher FEL frequencies correspond to positive values of $\hat{\Delta}$.

${ }^{3}$ Note that both $V_{n}$ and $S_{n}$ are functions of $\Delta$.
}

components, and the system has $N$ eigenvectors $V_{n}$, $n=1, \ldots, N$. We refer to these linearly independent solutions as "eigenmodes." Thus, in a general case the solution is a linear superposition of either finite or infinite number of eigenmodes:

$$
V(z)=\sum_{n=1}^{N \text { or } \infty} V_{n} e^{s_{n} z}
$$

which evolve along $z$ with exponential factors of $e^{s_{n} z}$, where $\left\{s_{n}\right\}$ are the eigenvalues of the corresponding dispersion relation-Eq. (2). Since the solutions with positive real part $\operatorname{Re}\left(s_{n}\right)>0$ are growing exponentially, knowing their number and their growth rate is the key for any highgain FEL.

In this paper, we prove a 1D FEL driven by an e-beam with bell-shaped energy distribution has either one or no growing solution to which we will assign index $n=1$. This means that all other solutions with $n \neq 1$ either decay (i.e. $\operatorname{Re}\left(s_{n}\right)<0$ ) or oscillate without amplification [i.e. $\left.\operatorname{Re}\left(s_{n}\right)=0\right]$. It also means that in a high-gain FEL with $G=\left|e^{s_{n} L}\right| \gg 1$, where $L$ is the FEL length, the solution (4) can be approximated by a single term

$$
V(z) \cong V_{1} e^{s_{1} z} .
$$

Importance of the latter can be demonstrated by a nontrivial example of the optical power evolution at the central wavelength $(\hat{\Delta}=0)$ in an FEL driven by electron beam with $\hat{\Lambda}_{p}=0.2 \kappa-2$ energy spread of $f(\hat{P})=\frac{2}{\pi\left(1+\hat{P}^{2}\right)^{2}}$. Such a FEL has four eigenmodes, with only one of them growing. As seen in Fig. 1, the growing mode completely dominates the remaining modes after two gain length.

For most short wavelength FELs the longitudinal space charge does not play an important role and we neglect it in

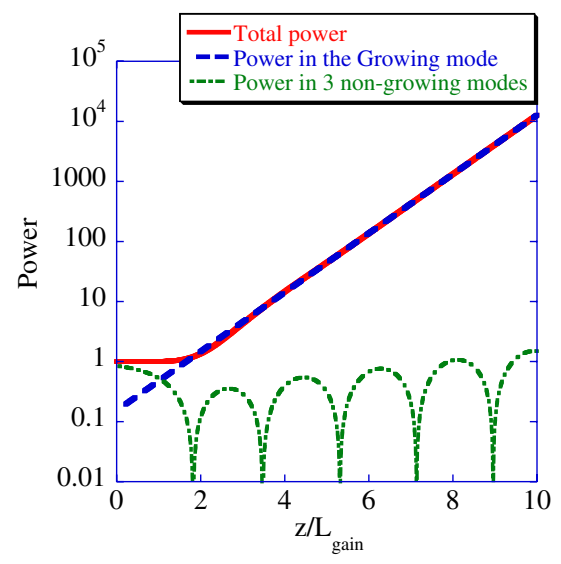

FIG. 1. Evolution of FEL optical power along the FEL: solid line (red) - the total power in all four eigenmodes; dashed line (blue) - the power in the growing eigenmode; dotted line (green)_remaining power in three nongrowing modes. Horizontal axis-length along the FEL in the units of gain length. Vertical axis in log-scale-optical power in units of the input power. 
(a)

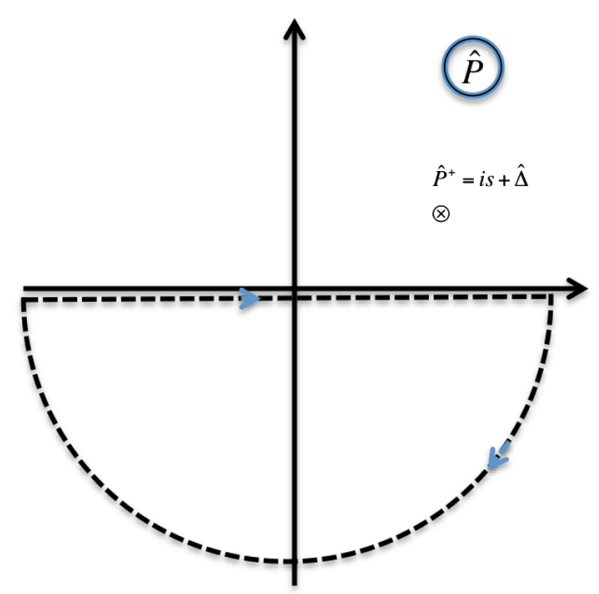

(b)

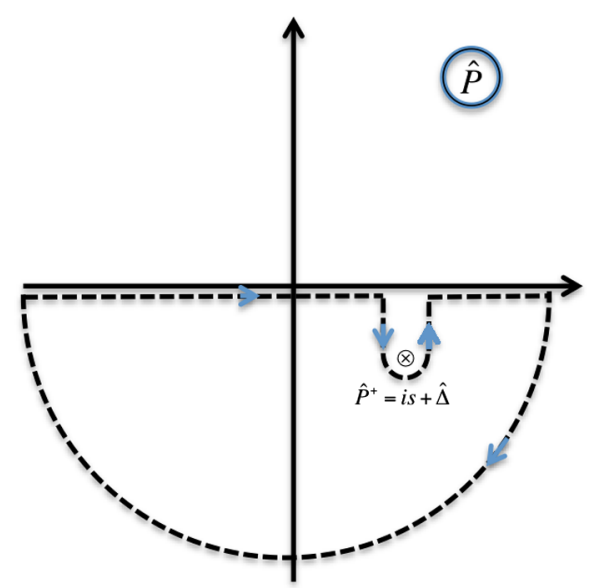

FIG. 2. (a) The integration contour for the evaluation of $\hat{D}(s)$ at $\operatorname{Re}(s)>0$. (b) The integration contour for the analytical continuation of $\hat{D}(s)$ into $\operatorname{Re}(s) \leq 0$.

this paper, ${ }^{4}$ we therefore focus on the reduced dispersion equation $w(s)=0$, where

$$
w(s)=s-\hat{D}(s) .
$$

In this paper, we therefore focus on the reduced dispersion relation (6).

\section{CORE OF THE METHOD}

Let us start from exploring some basic features of the dispersion function $\hat{D}(s)$, especially the location of its poles. When evaluating the Laplace transform of the linear FEL equation [12],

$$
\mathcal{L}[f(t)]=\int_{0}^{\infty} d t e^{-s t} f(t),
$$

it is assumed that $\operatorname{Re}(s)>0$ by the consideration of causality and the convergence of the above integral. Corresponding contours in the complex $\hat{P}$ plane for the evaluation of Eq. (3) for $\hat{D}(s)$ in the $\operatorname{Re}(s)>0$ half-plane is shown in Fig. 2(a). Figure 2(b) shows the contour for the correct analytic continuation of $\hat{D}(s)$ to the $\operatorname{Re}(s) \leq 0$ halfplane, as first shown in [16].

In all cases the pole at $\hat{P}=i s+\hat{\Delta}$ remains above the contour of integration and $\hat{D}(s)$ is nonzero function only if $\hat{F}^{\prime}$ has poles $\hat{P}_{n}^{*}$ located in the lower complex plane $\left[\operatorname{Im}\left(\hat{P}_{n}^{*}\right)<0\right]$. Then evaluating Eq. (3) using the residue formula will generate poles in $\hat{D}(s) \mathrm{at}^{5}$

\footnotetext{
${ }^{4}$ It also makes mathematics more tractable in this short paper. Otherwise the logic is similar but should be applied to the following function: $w(s)=s\left[1-i \hat{\Lambda}_{p}^{2} \hat{D}(s)\right]-\hat{D}(s)$.

${ }^{5}$ For illustration, a well-known rule $\oint\left[a_{n} /\left(\hat{P}-\hat{P}_{n}^{*}\right)\right]$. $\{d \hat{P} /[s+i(\hat{P}-\hat{\Delta})]\}=2 \pi i a_{n} /\left[s+i\left(\hat{P}_{n}^{*}-\hat{\Delta}\right)\right]$, where the integral is taken around the pole $\hat{P}_{n}^{*}$, shows that a simple pole of $\hat{F}^{\prime}$ located at $\hat{P}_{n}^{*}$ does generate a simple pole in $\hat{D}(s)$ located at $s=$ $-i\left(\hat{P}_{n}^{*}-\hat{\Delta}\right)$.
}

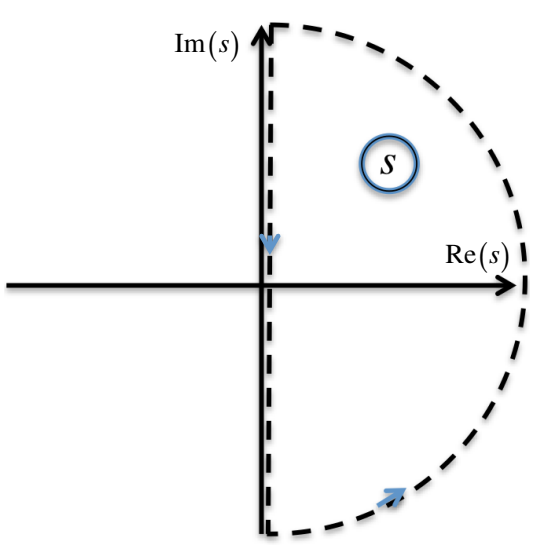

FIG. 3. The contour to determine the winding number encompasses the right half-plane.

$$
s_{n}^{*}=-i\left(\hat{P}_{n}^{*}-\hat{\Delta}\right) .
$$

Since $\operatorname{Im}\left(\hat{P}_{n}^{*}\right)<0$, we concluded that $\operatorname{Re}\left(s_{n}^{*}\right)=\operatorname{Im}\left(\hat{P}_{n}^{*}\right)<0$, i.e. all poles of $\hat{D}(s)$ are located in the left half-plane, $\operatorname{Re}(s)<0$.

The above analytical features of the dispersion function allow the argument principle of complex analysis to determine the number of roots of the dispersion relation (6) using the contour $C$ shown in Fig. 3 and the fact that the change in the argument of a complex function $w(s)$ meromorphic on a closed contour $C$, is given by its winding number,

$$
W=Z-P=\frac{1}{2 \pi} \oint d\{\arg [w(s)]\},
$$

where $Z$ is the number of zeros of $w(s)$ within the contour $C$ and $P$ is the number of poles, counting multiplicity. This 
is a direct analogy of the Nyquist stability criterion [17,18] developed for the analysis of linear circuits.

Since the poles of $w(s)=s-\hat{D}(s)$ are defined by that of $\hat{D}(s)$, there are no poles inside the contour $C$ and $P \equiv 0$. Hence, the winding number is equal to the number of roots of the dispersion relation (3) with $\operatorname{Re}\left(s_{n}\right)>0$, and therefore to that of the growing solutions.

Let us separate the contour $C$ in two parts: the $\operatorname{arc} s=$ $R \cdot e^{i \theta} ;-\pi / 2+\arcsin (\varepsilon / R)<\theta<\pi / 2+\arcsin (\varepsilon / R)$ and the vertical line $s=\varepsilon+i \xi,-R<\xi<R$ with $R \rightarrow \infty$ with $\varepsilon$ being an arbitrary small positive number. As shown in Appendix A, for a variety of physical energy distributions the ratio of $\hat{D}(s) / s \rightarrow 0$ at the arc when $R \rightarrow \infty$. It means that the change of the argument along the arc is determined by that of $s=R \cdot e^{i \theta}: \int_{\operatorname{arc}} d\{\arg [w(s)]\}=\pi$. It also means that the arcs in $s$ and $w$ complex planes are identical. The determination of the number of roots is therefore entirely determined by the behavior of the vertical component of the contour.

We determine the change of the argument along the vertical axis by evaluating the dispersion integral (3) at $s=i \xi, \xi \in\{-\infty, \infty\}$. Applying a well-known identity

$$
[\cdots] \frac{1}{x} d x=\mathcal{P}\left([\cdots] \frac{1}{x}\right) d x+i \pi[\cdots] \delta(x) d x,
$$

where $\mathcal{P}$ indicates the Cauchy principal value and $\delta(x)$ is Dirac's $\delta$ function, to Eq. (3) we get

$$
\begin{aligned}
\hat{D}(i \xi) & =\int \frac{d \hat{F}}{d \hat{P}} \frac{d \hat{P}}{i(\hat{P}+\xi-\hat{\Delta})} \\
& =-i \mathcal{P} \int \frac{d \hat{F}}{d \hat{P}} \frac{d \hat{P}}{(\hat{P}+\xi-\hat{\Delta})}+\pi \hat{F}^{\prime}(\hat{\Delta}-\xi) .
\end{aligned}
$$

As we can see in the following sections, the shape of the energy distribution function determines topology of the contour and the later determines the winding number and, as the result, the number of the growing modes.

\section{BELL-SHAPED ENERGY DISTRIBUTIONS}

We define a bell-shaped energy distribution as a smooth, positive, vanishing at infinity, bounded function with a single maximum. In this chapter, without loss of generality, we assume that the maximum of our energy distribution function is located at $\hat{P}=0$, setting $\hat{F}^{\prime}(0)=0$. For the purpose of clarity, we note that for such a bell-shaped distribution $\hat{F}^{\prime}(x>0) \leq 0$ and $\hat{F}^{\prime}(x<0) \geq 0$.

From Eq. (9), one can see that along the vertical axis $s=$ $i \xi, \operatorname{Re} w(i \xi)=-\pi \hat{F}^{\prime}(\hat{\Delta}-\xi)$. Because $\hat{F}^{\prime}(\xi-\hat{\Delta})=0$ has only one root at $\xi=\hat{\Delta}$, the map of the contour $C$ onto $w$ complex plane crosses imaginary axis $\operatorname{Re} w=0$ only once. Since we assumed that $\hat{F}(\hat{P})$ is bounded and positive [i.e. $\hat{F}(x) \geq 0 ; \hat{F}(x)_{|x| \rightarrow \infty} \rightarrow 0$ ], $\hat{\Delta}$ is a finite number, the sign of the derivative at the extremes of the imaginary axis are also fully determined: the real part of $w(i \xi)$ is positive when $\xi \rightarrow-\infty$ and is negative when $\xi \rightarrow \infty$. To be exact, the following is true:

$$
\begin{array}{ll}
\operatorname{Re} w(i \xi)=-\hat{F}^{\prime}(\hat{\Delta}-\xi) \geq 0 ; & \xi<\hat{\Delta} \\
\operatorname{Re} w(i \xi)=-\hat{F}^{\prime}(\hat{\Delta}-\xi) \leq 0 ; & \xi>\hat{\Delta} .
\end{array}
$$

Therefore, the contour $w(i \xi)$ crosses from the right half-plane to the left half-plane when $\xi$ increases. Thus, topologically there are only three options for the contour $w(C)$, which are shown in Figs. 4(a)-4(c), i.e. the crossing occurs above, on, or below the real axis $\operatorname{Im}(w)=0$. In the first case, $W=1$ and there is a single growing solution. In the third case, $W=0$ and there is no growing solutions. The second case corresponds to the boundary between the two ranges (see below). This concludes the proof that FELs driven by an electron beam with a bell-shaped energy distribution have two ranges of frequencies. In the first range, there is one (and only one) growing solution. In the other, all solutions are either decaying or oscillating. (a)

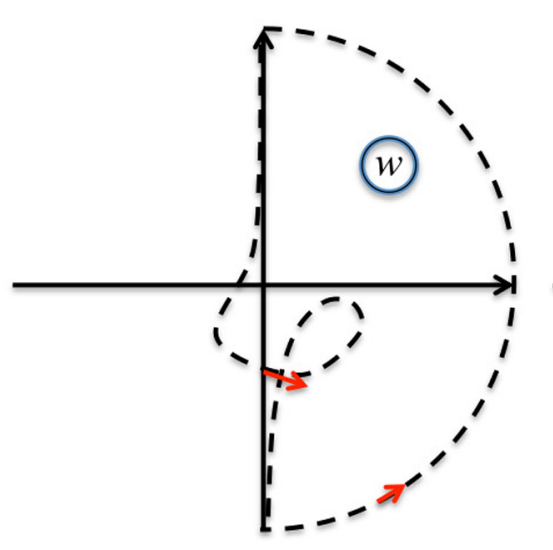

(b)

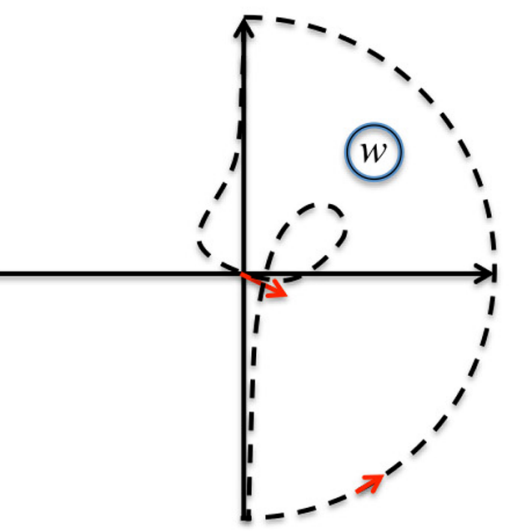

(c)

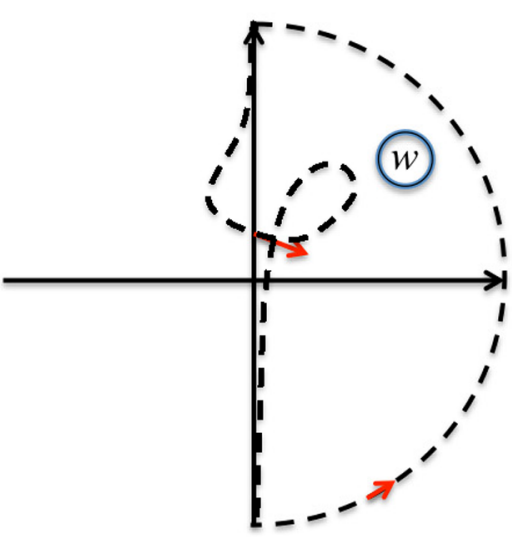

FIG. 4. (a) Map of the contour $C$ corresponding to one growing eigenmode, $W=1$. (b), (c) Map of the contour $C$ corresponding to the absence of the growing eigenmodes. 
It is worth noticing that local loops, which are not only possible but even quite frequent in the topology of practical $w(C)$ contours, cannot go around the origin without a second crossing of the axis, which is prohibited by the assumption of a simple maxima in the energy distribution.

This completes our proof that bell-shaped energy distributions can support one or no growing modes in an FEL, depending on the detuning from the FEL resonance.

Since $\hat{\Delta}$ is the only free parameter in (9), its value determines the boundary between these two ranges of frequencies. As illustrated in Fig. 4(b), the boundary is reached when the contour $w(C)$ crosses the origin $w=0$. First, the real part is equal to zero, i.e. $\xi=\hat{\Delta}$. Second, the imaginary part of (6) is also equal to zero, giving us the equation for the cutoff frequency $\hat{\Delta}^{+}$. Plugging $\xi=\hat{\Delta}$ into Eq. (9) we get

$$
w\left(i \hat{\Delta}^{+}\right)=i \hat{\Delta}^{+}+i \mathcal{P} \int \frac{d \hat{F}}{d \hat{P}} \frac{d \hat{P}}{(\hat{P})}+\pi \hat{F}^{\prime}(0)=0
$$

and $\hat{F}^{\prime}(0)=0$ yields us the value of the maximum detuning from the FEL resonance to be

$$
\hat{\Delta}^{+}=-\int \frac{d \hat{F}}{d \hat{P}} \frac{d \hat{P}}{\hat{P}} .
$$

One should notice that for the bell-shaped distribution $\hat{\Delta}^{+} \geq 0$, which means that the amplification vanishes at high frequencies, while such FELs will amplify all low frequencies. Hence, Eq. (10) determines analytically the cutoff frequency of the FEL. This equation can be easily evaluated for any specific case. For example, the Gaussian energy distribution of $\hat{F}(\hat{P})=\exp \left\{-\hat{P}^{2} / 2 \sigma^{2}\right\} / \sqrt{2 \pi} \sigma^{2}$ yields

$$
\hat{\Delta}^{+}=\frac{1}{\sigma^{2}} .
$$

Table I gives expressions for $\hat{\Delta}^{+}$for some bell-shaped distributions. ${ }^{6}$ Hence, of other bell-shaped distributions with typical energy spread of $\sigma$, one can estimate the cutoff to be at $\hat{\Delta}^{+} \propto \sigma^{-2}$ with a numerical coefficient on the order of unity and dependent on the particulars of $\hat{F}(\hat{P})$.

Since the Lorentzian distributions have infinite rms spread value, the identical measure of $\sigma$ is hard to be found, except the FWHM values which are $2 \sigma, 2 \sqrt{2} \sigma$, $4 \sqrt{\sqrt{2}-1} \sigma, 2 \sqrt{6(\sqrt[3]{2}-1)} \sigma, 2 \sqrt{2 n(\sqrt[n]{2}-1)} \sigma, \sqrt{2 \ln 2} \sigma$, correspondingly. It is also possible to frame the $\kappa-n$ distributions as a limit converging to a Gaussian, which then means that in the large $n$ limit, the kappa distributions

\footnotetext{
${ }^{6}$ There is one notable exception-for the monoenergetic (delta-function) energy distribution $\sigma=0$ and there is no high-frequency cutoff. It is rather interesting that the singularity of such energy distribution creates an unusual low-frequency cutoff at $\hat{\Delta} \cong-1.9$ (as shown in Fig. 2.2, page 26 in Ref. [12]). This cutoff does not appear for any smooth distribution functions.
}

TABLE I. Cutoff frequency of FEL with bell-shaped energy distributions.

\begin{tabular}{llc}
\hline \hline $\begin{array}{l}\text { Distribution } \\
\text { function }\end{array}$ & \multicolumn{1}{c}{ Formula } & $\hat{\Delta}^{+}$ \\
\hline Rectangular & $F(x)=\frac{1}{2 \sigma} ;|x| \leq \sigma$ & $\frac{1}{\sigma^{2}}$ \\
Lorentzian & $F(x)=\frac{1}{\sqrt{2} \pi \sigma\left(1+x^{2} / 2 \sigma^{2}\right)}$ & $\frac{1}{2} \cdot \frac{1}{\sigma^{2}}$ \\
$\kappa-2$ & $F(x)=\frac{1}{\pi \sigma\left(1+x^{2} / 4 \sigma^{2}\right)^{2}}$ & $\frac{3}{4} \cdot \frac{1}{\sigma^{2}}$ \\
$\kappa-3$ & $F(x)=\frac{4 \sqrt{2}}{3 \sqrt{3} \frac{1}{\pi \sigma\left(1+x^{2} / 6 \sigma^{2}\right)^{3}}}$ & $\frac{5}{6} \cdot \frac{1}{\sigma^{2}}$ \\
$\kappa-n$ & $F(x)=\frac{\Gamma(n)}{\Gamma(n-1 / 2)} \frac{1}{\sqrt{2 \pi n} \sigma\left(1+x^{2} / 2 n \sigma^{2}\right)^{n}}$ & $\frac{2 n-1}{2 n} \cdot \frac{1}{\sigma^{2}}$ \\
Gaussian & $\hat{F}(x)=\frac{1}{\sqrt{2 \pi} \sigma} e^{-x^{2} / 2 \sigma^{2}}$ & $\frac{1}{\sigma^{2}}$ \\
\hline \hline
\end{tabular}

will converge to a Gaussian with variance $\sigma$. Thus, in the absence of convergent moments, we may understand the energy spread for $\kappa-n$ distributions in terms of the welldefined Gaussian parameter.

To summarize this result, assuming that the 1D FEL is driven by an electron beam with a smooth, positive, bounded energy distribution function with a single maximum, going to zero at $\hat{P} \rightarrow \pm \infty$, we proved that there is only a single growing FEL eigenmode, which amplifies the radiation at frequencies below the cutoff value. The rest of the eigenmodes are either decaying or oscillating. Above the cutoff frequency there are no growing solutions and all of them are either decaying or oscillating. We also derived a simple analytical formula for the frequency cutoff in the form of one-dimensional integral, which can be easily evaluated either analytically (as we did above for a Gaussian energy distribution) or numerically.

\section{GENERAL CASE}

Let us consider a general case of energy distribution described by a smooth, positive, vanishing at infinity, bounded function. We are no longer making any assumptions about number of maxima and minima, nor about their location. The assumption of a smooth vanishing at infinity distribution function is that the number of maxima is equal to that of minima plus one. Let us assume an energy distribution with $N$ local maxima (and $N-1$ local minima).

As was shown in the previous section, when $\hat{F}^{\prime}(\hat{\Delta}-\xi)=0$ at a local extremum, the contour crosses the imaginary axis - the same argument is correct for a general energy distribution. Furthermore, it is easy to see that at maxima, where $\hat{F}^{\prime}(\hat{\Delta}-\xi)<0$, the contour crosses the imaginary axis from left to right [e.g. as in Figs. 4(a)-4(c)], and that at local minima, where $\hat{F}^{\prime \prime}(\hat{\Delta}-\xi)>0$ the contour crosses the imaginary axis from right to left when $-\xi$ changes from $-\infty$ to $\infty$, i.e. when we are going along the straight part of the contour $C$. With $N$ local maxima this can lead to as many as $N$ windings around the origin and as many as $N$ growing modes. Each mode can have cutoffs either from one or both sides. 
(a)

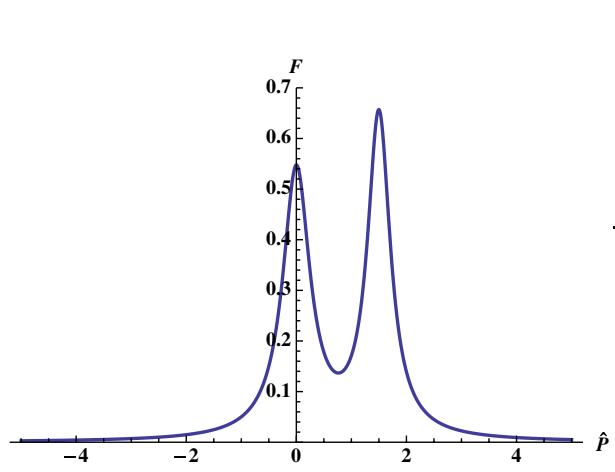

(b)

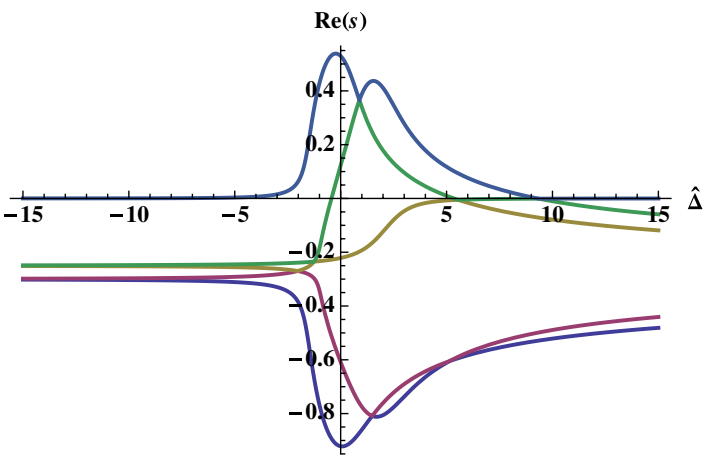

FIG. 5. (a) Bi-Lorentzian energy distribution with $A=0.5 ; \sigma_{1}=0.3, \sigma_{2}=0.25, \delta=1.5$. (b) Real parts of five roots of the dispersion relation (15) as functions of the detuning.

A more explicit calculation may be made to characterize the number of growing modes versus the detuning. The real part of the dispersion relation is given by

$$
\hat{F}^{\prime}\left(\hat{\Delta}-\xi_{i}\right)=0,
$$

where the detuning must be defined in reference to some characteristic energy, and $\xi_{i}$ characterizes some root.

Let us consider how windings appear and disappear topologically. Running from $\xi=-\infty$, the first extremum must be a local maximum given our assumptions. Denote its location by $\xi_{o}$. Now consider

$$
\operatorname{sgn}\left(\mathcal{P} \int \frac{d \hat{F}}{d \hat{P}} \frac{d \hat{P}}{\left(\hat{P}+\xi_{0}-\hat{\Delta}\right)}\right) .
$$

The total number of windings can be calculated by keeping track of how many times the contour crosses from the one half-plane to the other, and how. Details of this method can be found in Appendix B.

As an example, consider the following nontrivial normalized energy distribution with first maxima located at $\hat{P}=0$ :

$$
F(\hat{P})=\frac{A}{\pi} \frac{\sigma_{1}}{\hat{P}^{2}+\sigma_{1}^{2}}+\frac{1-A}{\pi} \frac{\sigma_{2}}{(\hat{P}-\delta)^{2}+\sigma_{2}^{2}},
$$

where $0 \leq A \leq 1 ; \sigma_{1,2}, \delta$ are parameters we can vary. The corresponding dispersion relation is a fifth-order polynomial and has five roots:

$$
\begin{aligned}
\hat{D}(s)= & A \frac{i}{\left(s+\sigma_{1}-i \hat{\Delta}\right)^{2}}+(1-A) \\
& \times \frac{i}{\left[s+\sigma_{2}-i(\hat{\Delta}-\delta)\right]^{2}} \\
= & s .
\end{aligned}
$$

According to our theorem, the maximum number of growing modes could not exceed two. The following examples show that depending of the choice of the parameter of the distribution function maximum number of growing modes can be either two or one. Choosing $A=0.5 ; \sigma_{1}=$ $0.3, \sigma_{2}=0.25, \delta=1.5$, we have the distribution function shown in Fig. 5(a), which, as it can be seen from Fig. 5(b), provides for the maximum of two growing modes in some range of detuning.

As it can be seen from the plot in Fig. 5(b) there is one growing mode at low frequencies $\hat{\Delta}<-0.436$; two growing modes at $-0.436<\hat{\Delta}<5.352$; one growing mode at $5.352<\hat{\Delta}<9.301$ and none above the cutoff $\hat{\Delta}>9.301$.

Plots in Fig. 6 show the corresponding contours at four characteristic detuning corresponding to this four case.

As one can see from Fig. 6, the winding number is equal to a corresponding number of growing roots. At a detuning $\hat{\Delta}=-1$ there is one growing mode, and in Fig. 5(a), we see that the contour winds around the origin once. At $\hat{\Delta}=+1$, we observe two simultaneous growing modes, and indeed there are two windings with the second local loop (winding) around the origin [Fig. 6(b)]. The first mode stops amplifying, leaving only the second mode at $\hat{\Delta}=3$, as can be seen in Fig. 6(c), and then there are no growing modes at $\hat{\Delta}=11$, Fig. $6(\mathrm{~d})$.

The most interesting case is shown in Fig. 6(b), where we can clearly see the second local loop (winding) around the origin.

We had shown all typical cases of contours in Fig. 6 to compare them with the set of rules we developed in the paper and with exact numerical values for the roots of the dispersion equation, one can clearly notice that the change in the detuning appears as a vertical shift along the imaginary axis.

In fact, this feature of the contours is correct for an arbitrary case of 1D FEL dispersion relation (6). From Eqs. (3) and (6) one can easily prove the following identity:

$$
w(s)_{\Delta_{1}}=w(s)_{\Delta_{2}}+i \cdot\left(\Delta_{1}-\Delta_{2}\right),
$$

e.g. that a change of the detuning from $\Delta_{1}$ to $\Delta_{2}$ moves the contour along the vertical axis for $\Delta_{1}-\Delta_{2}$. Hence, a single contour is sufficient to determine the topology and 
(a)

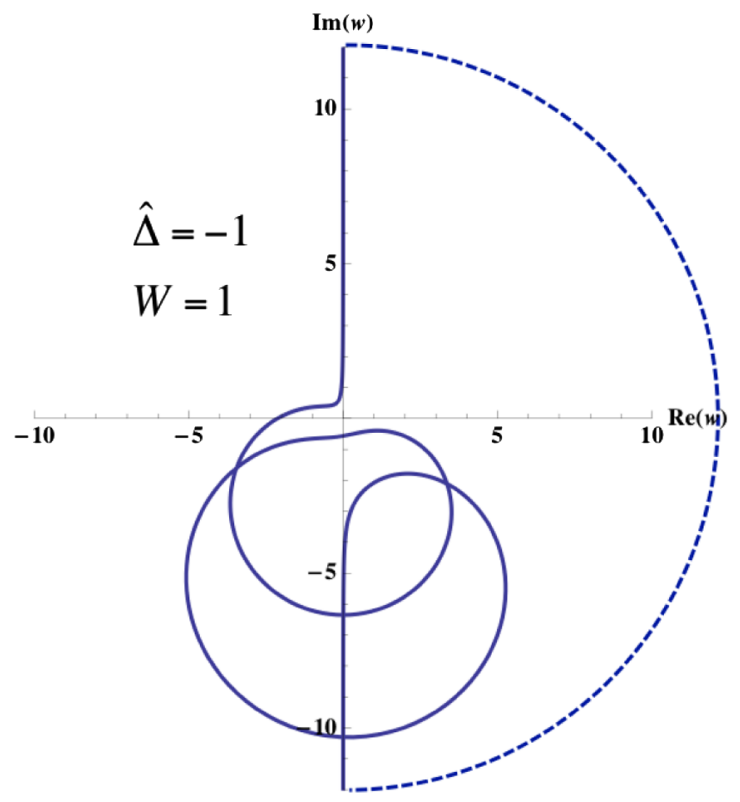

(c)

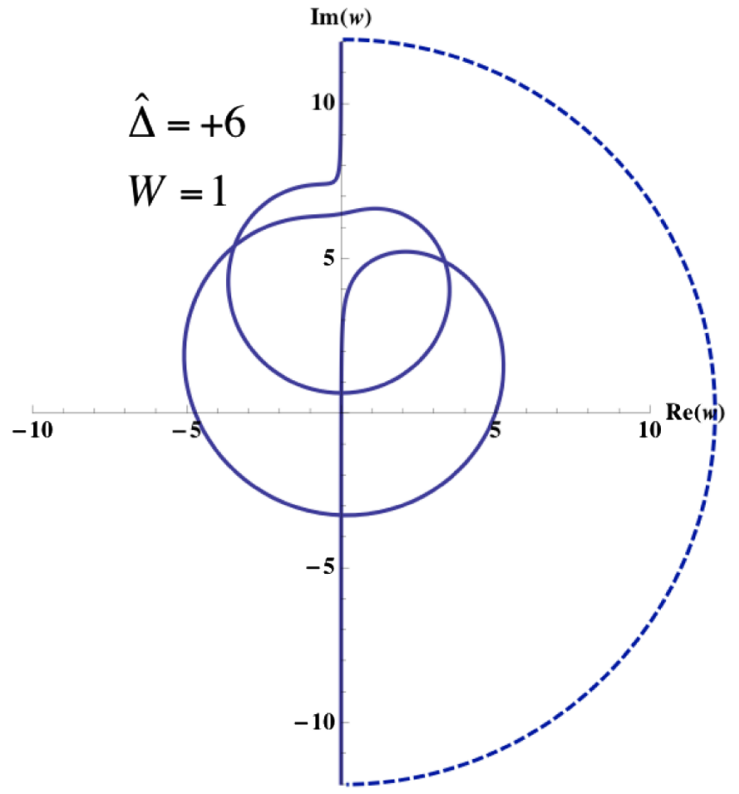

(b)

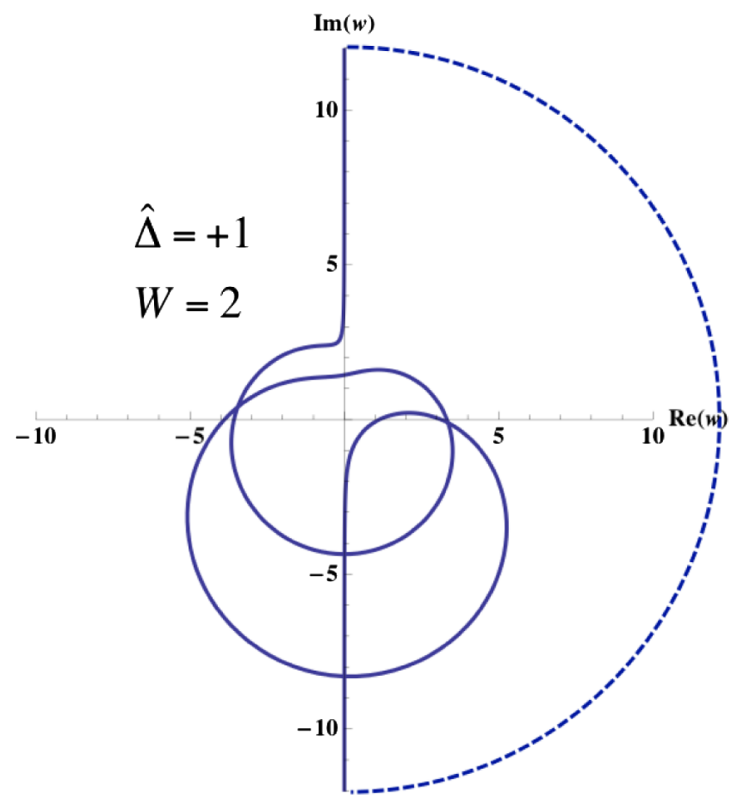

(d)

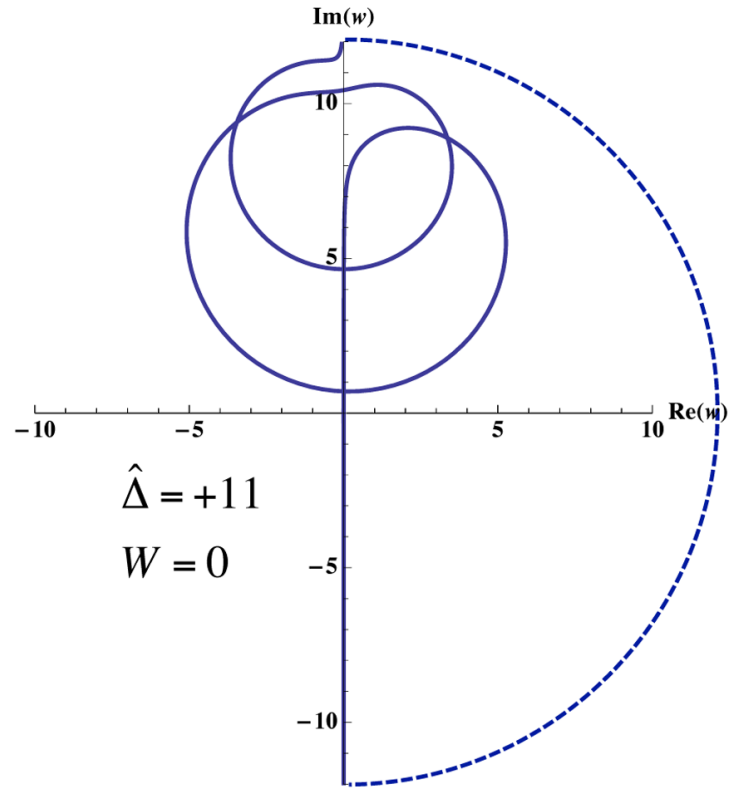

FIG. 6. Parts (a), (b), (c), and (d) are plots of $w(C)$ (with a dashed semicircle to be pushed to infinity) for various characteristic values for $\hat{\Delta}=-1,1,6$, and 11 correspondingly. The energy distribution is shown in Fig. 5(a).

the maximum winding number. The crossings of the $\Delta=0$ contour with the imaginary axis do determine the values (with opposite sign) of the detuning where the number of growing modes changes. Since the main loop is contraclockwise by design (i.e. has winding number plus one when it encircles the origin), the topology of the local loops depends on their helicity-if it encircles the origin, a clockwise reduces $W$ by one, while a contraclockwise increases $W$ by a unit. The direction is determined by the contour $C$ in Fig. 3.

Imagine $\hat{\Delta}$ being a vertical displacement of the origin. As one can see from Fig. 7, the local loop contraclockwise and pushing the origin into it would increase the winding number by one. One can easily see that winding number is equal to one at negative $\hat{\Delta}$, then at $\hat{\Delta}=-0.436$ the origin would move into the local loop and the winding number 


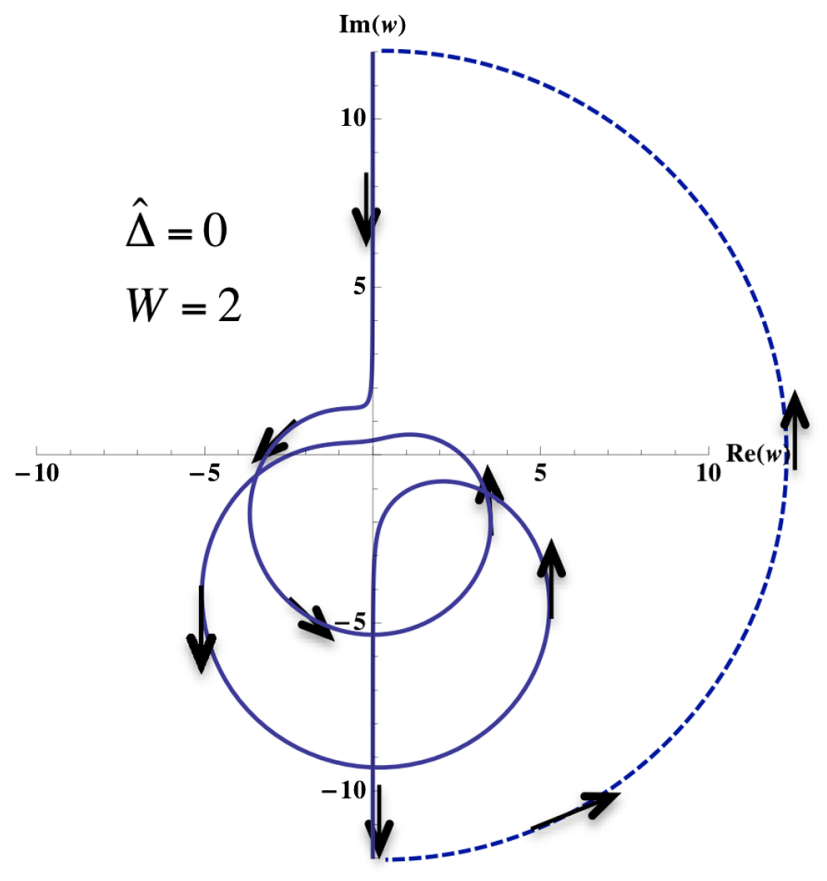

FIG. 7. Contour of $w(C)$ (with scaled down semicircle) for zero detuning $\hat{\Delta}=0$.

would increase to 2 . Similarly, at $\hat{\Delta}=5.352$, the origin moves out of the loop, reducing the winding number by a unit, and further reducing it to zero when the tuning goes above 9.301 and crosses the main loop.

Hence, using this simple rule, we consider another example of bi-Lorentzian energy distribution with $A=0.8$, $\sigma_{1}=1, \sigma_{2}=0.25, \delta=3$ (see Fig. 8), shows the energy distribution and real part of the roots as function of the detuning.

Figure 9 shows the contour plot for the energy distribution shown in Fig. 8(a). One can see that, in contrast with Fig. 7, the local loop does not cross the imaginary axis, and therefore does not affect the winding number. What is striking is that the main contour makes a brief detour into the right-hand side and causes an additional frequency

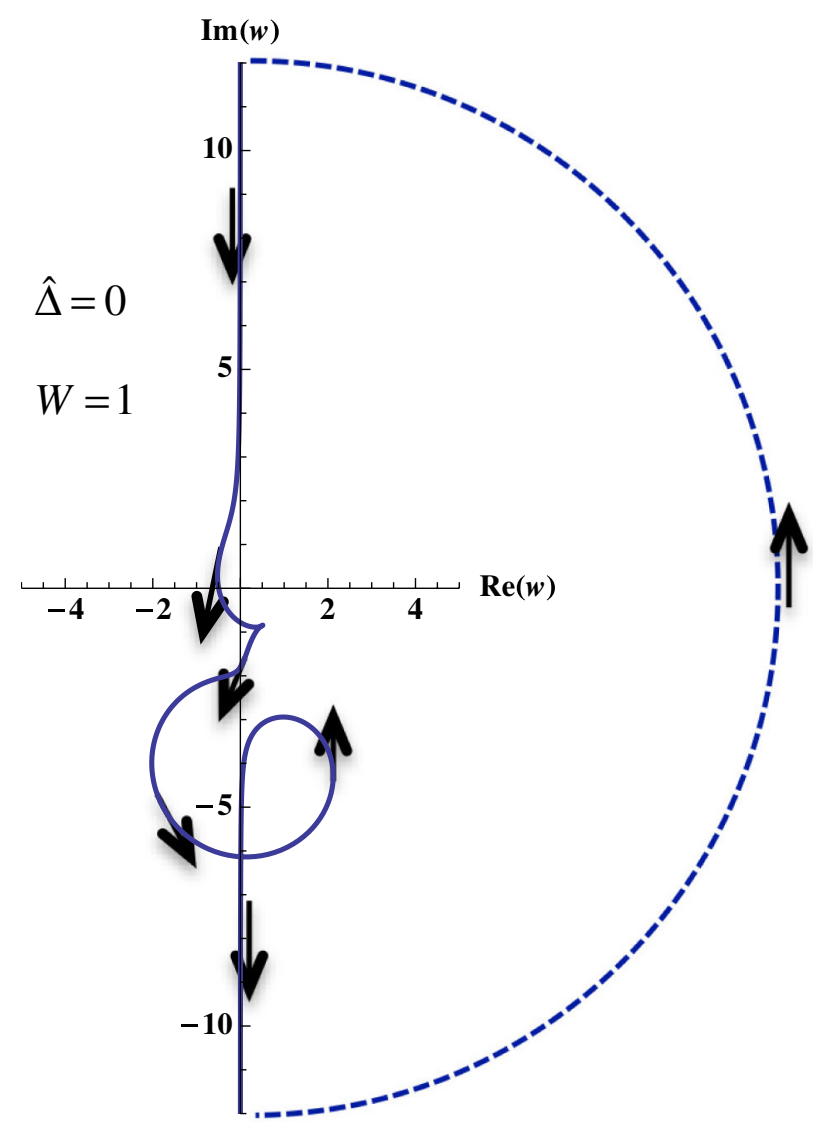

FIG. 9. Contour of $w(C)$ (with scaled down semicircle) for zero detuning $\hat{\Delta}=0$ and energy distribution shown in Fig. 8(a).

region where there are no growing modes. In short, there is one growing mode at low frequencies below $\hat{\Delta}=0.78$, where a dead-zone without growing modes extends to $\hat{\Delta}=1.805$. A second zone with a single growing mode $\hat{\Delta}>1.805$ continues to the high-frequency cutoff at $\hat{\Delta}=6.134$. Naturally these topological observations are in exact agreement with direct solutions of the dispersion relations, shown in Fig. 8(b), from where one can also see
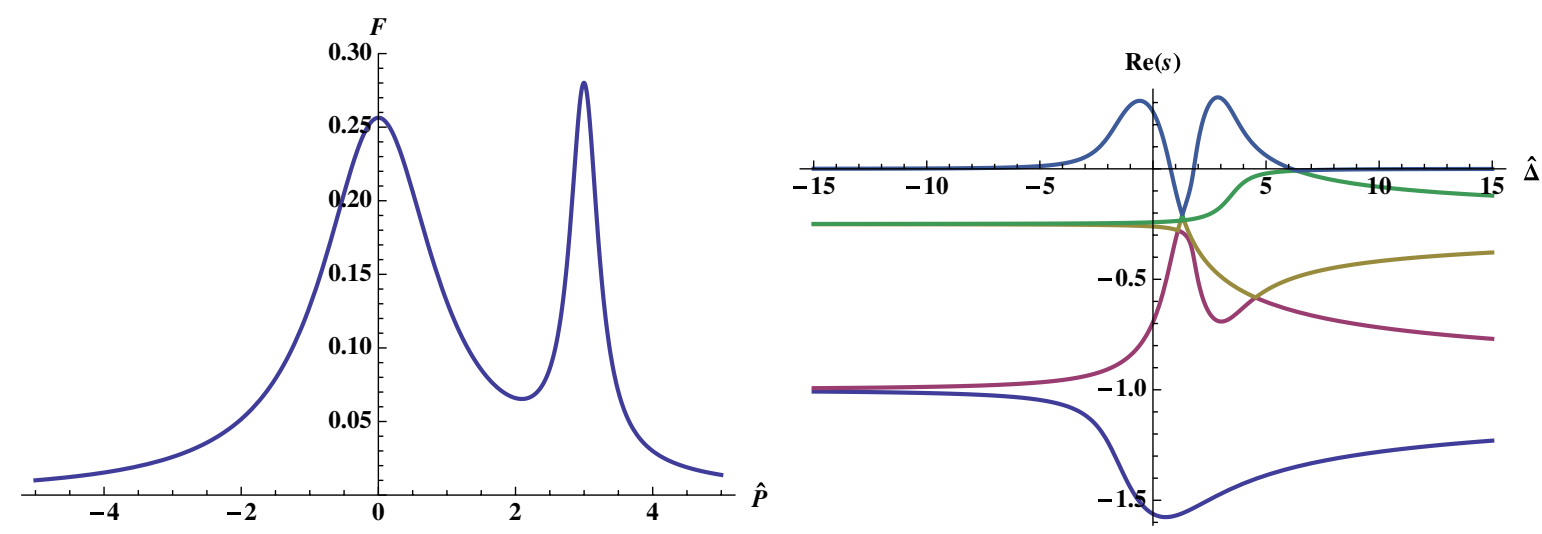

FIG. 8. (a) Bi-Lorentzian energy distribution with $A=0.8 ; \sigma_{1}=1, \sigma_{2}=0.25, \delta=3$. (b) Real parts of five roots of the dispersion relation (15) as functions of the detuning. 
(a)

(b)

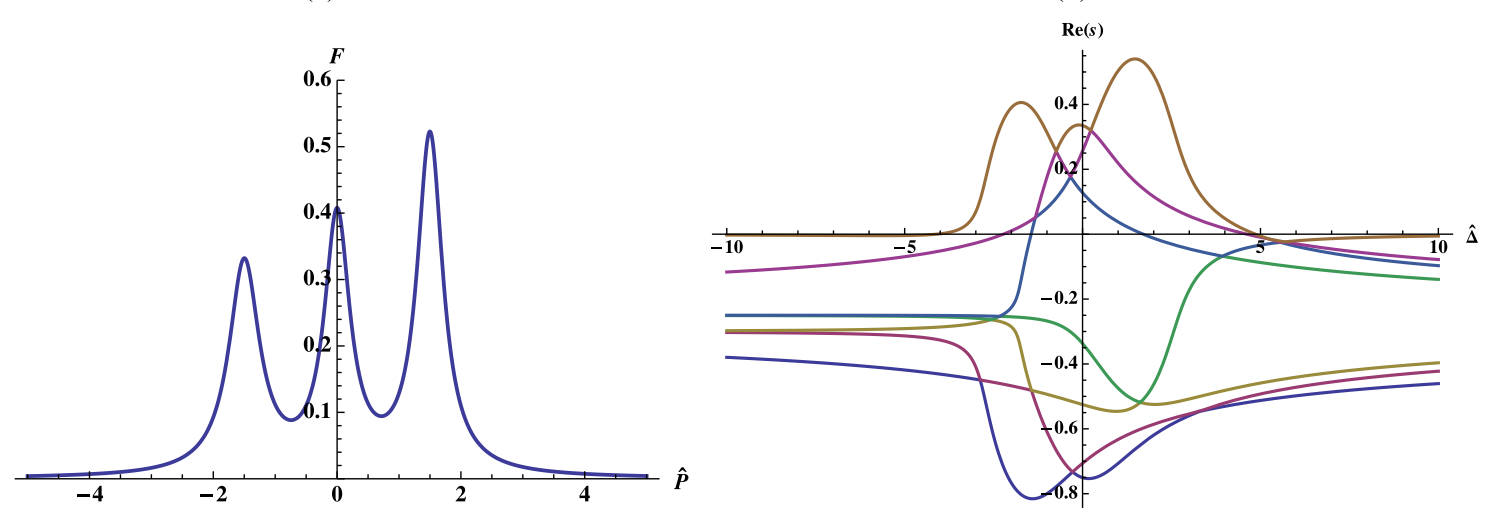

(c)

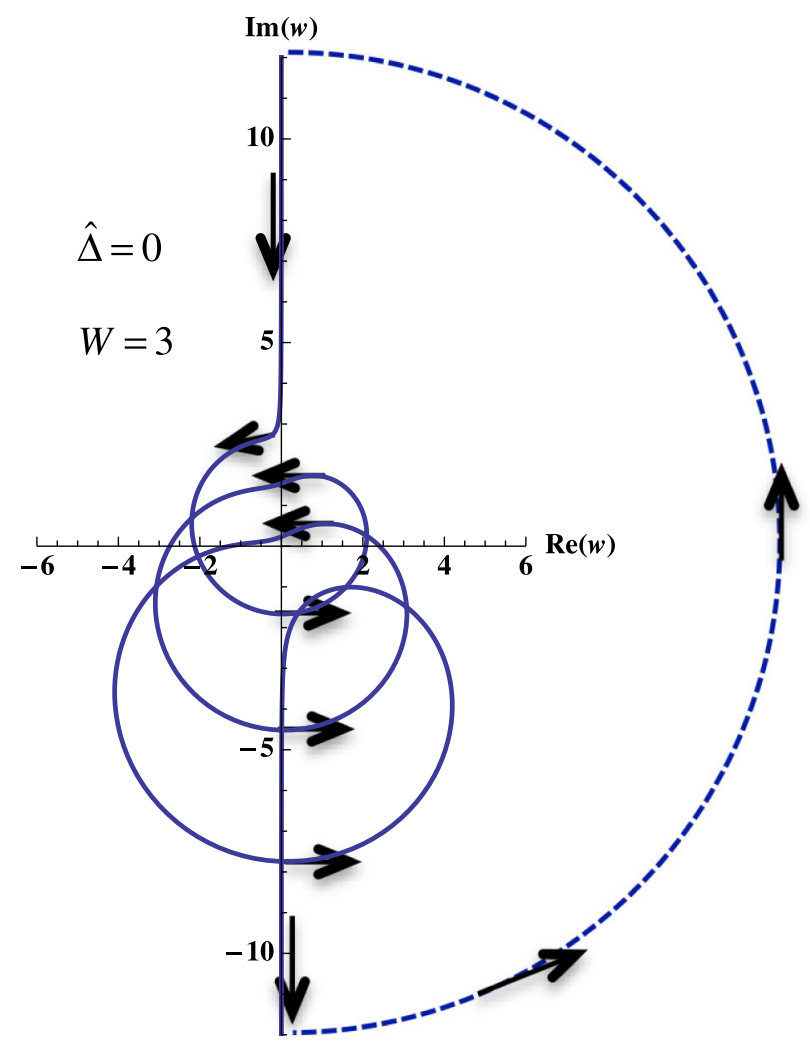

FIG. 10. (a) Tri-Lorentzian energy distribution. (b) Real parts of seven roots of the dispersion relation as functions of the detuning. (c) Contour of $w(C)$ (with scaled down semicircle) for zero detuning $\hat{\Delta}=0$.

that two growth zones correspond to two different root branches of the fifth-order polynomial.

We conclude this section with Fig. 10 illustrating a triLorentzian energy distribution (and corresponding contour and real part of root of the dispersion equation), which supports up to three growing modes.

\section{CONCLUSION}

In this paper we proved two main results for the spacecharge-free dispersion relation of 1D FELs. First, we proved that for a bell-shaped ( $a$ smooth, positive, bounded energy distribution function with a single maximum) energy distribution of the electron beam, we have shown that causality requires that such a FEL will have only a single growing mode with a high-frequency cutoff. We derived analytical expression for the cutoff frequency in the form of a simple integral. We evaluated this integral for Gaussian energy distribution. This proof puts to rest the question about how many growing modes can be supported by Gaussian or semi-Gaussian energy distributions.

Furthermore, we consider a more general case of multiple energy peaks. In this case, we conclude that for fixed frequency, an energy distribution with $N$ local maxima will have between zero and $N$ growing modes, and we derive 
criteria and the method of determining the number of growing modes and their cutoff frequencies and cutoff ranges. We also demonstrated that contour of the dispersion relation at zero detuning is sufficient to determine all frequency ranges where growing modes exist as well as the number of modes in each growing zone.

We would like to emphasize that we used a method analogous to the Nyquist's stability criterion $[17,18]$ and Penrose's treatment of a stationary plasma driven by an oscillating external electric field with frequency $\omega$ [19], and for general "growing waves" [20] or other plasma instabilities [21,22]. We applied this method to the FEL dispersion relation, which differs quite significantly from the above systems, and found new, previously unknown features of a very fundamental nature. This is not very surprising since we were using well-established fundamental principles enshrined into the Laplace transform and Landau's contour integration technique, and causality.

This particular method appears to be periodically rediscovered by physicists over the decades. Because the Laplace transform and Fourier transform are related by a Wick rotation, the same formal structure exists either looking at the initial value problem or at frequency responses.

\section{ACKNOWLEDGMENTS}

The work presented represents continuing efforts from affiliation with Department of Physics \& Astronomy, Stony Brook University, and Collider-Accelerator Department, Brookhaven National Laboratory. The authors would like to thank Michael Blaskiewicz (BNL) and Ilan Ben-Zvi (BNL) for helpful discussion, and Ron Ruth (SLAC) and Zhirong Huang (SLAC) for drawing attention to the applicability of the general case. This work was supported by Brookhaven Science Associates, LLC under Contract No. DE-AC02-98CH10886 with the U.S. Department of Energy.

\section{APPENDIX A}

Assuming the distribution function satisfying $\lim _{|\hat{P}| \rightarrow \infty} \hat{F}(\hat{P})=0$, the dispersion integral can be written, after integration by parts, as

$$
\hat{D}(s)=-i \int_{-\infty}^{\infty} F(\hat{P}) \frac{1}{(\hat{P}-\hat{\Delta}-i s)^{2}} d \hat{P},
$$

for $\operatorname{Re}(s)>0$ (Ref. [23]). Defining

$$
s \equiv R \cdot e^{i[\theta+(\pi / 2)]},
$$

the requirement of $\operatorname{Re}(s)>0$ leads to

$$
\sin \theta<0 .
$$

Inserting (A3) into (A2) and changing the integration variable to

$$
\hat{P}_{1} \equiv \frac{\hat{P}}{R}
$$

produces

$$
\begin{aligned}
\hat{D}(s) & =-i \int_{-\infty}^{\infty} \frac{\hat{F}(\hat{P})}{\left(\hat{P}-\hat{\Delta}+R \cdot e^{i \theta}\right)^{2}} d P \\
& =\frac{-i}{R} \int_{-\infty}^{\infty} \frac{\hat{F}_{1}\left(\hat{P}_{1}\right)}{\left(\hat{P}_{1}-\frac{\hat{\Delta}}{R}+\cos \theta+i \sin \theta\right)^{2}} d \hat{P}_{1},
\end{aligned}
$$

where

$$
\hat{F}_{1}\left(\hat{P}_{1}\right)=\hat{F}\left(\hat{P}_{1} R\right) .
$$

Explicitly writing Eq. (A5) into real and imaginary part and change the integration variable to

$$
\hat{P}_{2} \equiv \hat{P}_{1}-\frac{\hat{\Delta}}{R}+\cos \theta
$$

Eq. (A5) becomes

$$
\hat{D}(s)=\frac{-1}{R} \int_{-\infty}^{\infty} \frac{\hat{F}_{2}\left(\hat{P}_{2}\right)\left(2 \hat{P}_{2} \sin \theta+i\left[\hat{P}_{2}^{2}-\sin ^{2} \theta\right]\right)}{\left(\hat{P}_{2}^{2}+\sin ^{2} \theta\right)^{2}} d \hat{P}_{2}
$$

with

$$
\hat{F}_{2}\left(\hat{P}_{2}\right)=\hat{F}_{1}\left(\hat{P}_{2}+\frac{\hat{\Delta}}{R}-\cos \theta\right)=\hat{F}\left[\left(\hat{P}_{2}+\frac{\hat{\Delta}}{R}-\cos \theta\right) R\right] .
$$

Taking into account Eq. (A3) and changing the integration variable of (A8) to

$$
x \equiv \frac{\hat{P}_{2}}{-\sin \theta}
$$

generates

$$
\hat{D}(s)=\frac{1}{R \sin \theta} \int_{-\infty}^{\infty} \frac{\hat{F}_{3}(x)\left(-2 x+i\left[x^{2}-1\right]\right)}{\left(x^{2}+1\right)^{2}} d \hat{P}_{3},
$$

with

$$
\hat{F}_{3}(x)=\hat{F}_{2}(-x \sin \theta)=\hat{F}\left[-\left(x \sin \theta-\frac{\hat{\Delta}}{R}+\cos \theta\right) R\right] .
$$

The amplitude of Eq. (A11) is given by

$$
\begin{aligned}
|\hat{D}(s)|^{2}= & \frac{1}{R^{2} \sin ^{2} \theta}\left\{\left[\int_{-\infty}^{\infty} \frac{\hat{F}_{3}(x) 2 x}{\left(x^{2}+1\right)^{2}} d x\right]^{2}\right. \\
& \left.+\left[\int_{-\infty}^{\infty} \frac{\hat{F}_{3}(x)\left(x^{2}-1\right)}{\left(x^{2}+1\right)^{2}} d x\right]^{2}\right\} .
\end{aligned}
$$

Noticing the distribution function $\hat{F}_{3}(x)=\hat{F}(\hat{P}) \geq 0$, $2 x \leq x^{2}+1$, and 


$$
\begin{aligned}
& {\left[\int_{-\infty}^{\infty} \frac{\hat{F}_{3}(x) x^{2}}{\left(x^{2}+1\right)^{2}} d x-\int_{-\infty}^{\infty} \frac{\hat{F}_{3}(x)}{\left(x^{2}+1\right)^{2}} d x\right]^{2}} \\
& \quad \leq\left[\int_{-\infty}^{\infty} \frac{\hat{F}_{3}(x) x^{2}}{\left(x^{2}+1\right)^{2}} d x+\int_{-\infty}^{\infty} \frac{\hat{F}_{3}(x)}{\left(x^{2}+1\right)^{2}} d x\right]^{2},
\end{aligned}
$$

we obtain the upper limit of $|D(s)|$ :

$$
|\hat{D}(s)|^{2} \leq \frac{2}{R^{2} \sin ^{2} \theta}\left[\int_{-\infty}^{\infty} \frac{\hat{F}_{3}(x)}{x^{2}+1} d x\right]^{2}
$$

i.e.

$$
|\hat{D}(s)| \leq \frac{\sqrt{2}}{R|\sin \theta|} \int_{-\infty}^{\infty} \frac{\hat{F}_{3}(x)}{x^{2}+1} d x
$$

Assuming the distribution function $\hat{F}(\hat{P})$ satisfies

$$
\hat{F}(\hat{P}) \leq a \hat{F}_{0}(\hat{P}), \quad \forall \hat{P}
$$

with

$$
\hat{F}_{0}(\hat{P}) \equiv \frac{1}{\pi \hat{q}} \frac{1}{1+\hat{P}^{2} / \hat{q}^{2}}
$$

and $a$ being the ratio of the maximum of $\hat{F}(\hat{P})$ with respect to $\hat{F}_{0}(\hat{P})$, i.e.

$$
a \equiv \frac{\hat{F}_{\max }}{\hat{F}_{0, \max }} .
$$

Applying Eq. (A17) to Eq. (A18) leads to

$$
\begin{aligned}
|\hat{D}(s)| \leq & \frac{\hat{F}_{\max } \sqrt{2}}{R|\sin \theta|} \int_{-\infty}^{\infty} \frac{1}{x^{2}+1} \frac{\hat{q}^{2}}{\hat{q}^{2}+\left(x \sin \theta-\frac{\hat{\Delta}}{R}+\cos \theta\right)^{2} R^{2}} d x \\
= & \frac{\sqrt{2} \pi \hat{F}_{\max } \tilde{q}^{2}}{R|\sin \theta|}\left\{\frac{1}{\left(i-\frac{\hat{\Delta}}{R \sin \theta}+\frac{\cos \theta}{\sin \theta}\right)^{2}+\tilde{q}^{2}}\right. \\
& \left.+\frac{1}{\tilde{q}} \frac{1}{\left(i \tilde{q}+\frac{\hat{\Delta}}{R \sin \theta}-\frac{\cos \theta}{\sin \theta}\right)^{2}+1}\right\} \\
= & \frac{\sqrt{2} \hat{F}_{\max } \pi \tilde{q}}{R|\sin \theta|} \frac{1+\tilde{q}}{\left(\frac{\cos \theta}{\sin \theta}-\frac{\hat{\Delta}}{R \sin \theta}\right)^{2}+(1+\tilde{q})^{2}}
\end{aligned}
$$

with

$$
\tilde{q} \equiv \frac{q}{R|\sin \theta|} .
$$

Inserting Eq. (A21) into Eq. (A20) leads to

$$
|\hat{D}(s)| \leq \sqrt{2} \pi \hat{q} \hat{F}_{\max } \frac{1}{R^{2}} \frac{1+\frac{\hat{q}}{R|\sin \theta|}}{\left(\cos \theta-\frac{\hat{\Delta}}{R}\right)^{2}+\left(\sin \theta+\frac{\hat{q}}{R}\right)^{2}} .
$$

In the limit of $R=|s| \rightarrow \infty$, Eq. (A22) becomes

$$
\lim _{|s| \rightarrow \infty}|\hat{D}(s)| \leq \sqrt{2} \pi \hat{q} \hat{F}_{\max } \frac{1}{|s|^{2}}\left[1+\frac{\hat{q}}{\operatorname{Re}(s)}\right]=0
$$

for $\operatorname{Re}(s)>0$.
Let us separate the contour $C$ in Fig. 3 in two homogenous parts, the $\operatorname{arc} s=R \cdot e^{i \theta} ;-\pi / 2+\arcsin \left(\frac{\varepsilon}{R}\right)<\theta<$ $\pi / 2+\arcsin \left(\frac{\varepsilon}{R}\right)$ and the vertical line $s=\varepsilon-i \xi-R<$ $\xi<R$ with $R \rightarrow \infty$. Let us consider $\varepsilon>0$ being an arbitrary small positive number. From Eq. (A23) we then can estimate that on the arc

$$
\lim _{|s| \rightarrow \infty}|\hat{D}(s)| \leq \sqrt{2} \pi \hat{q} \hat{F}_{\max } \frac{1}{|s|^{2}}\left[1+\frac{\hat{q}}{\operatorname{Re}(s)}\right]=0 .
$$

Selecting $R=\sqrt{\frac{\sqrt{2} \pi \hat{q} \hat{F}_{\max }}{\varepsilon}\left[1+\frac{\hat{q}}{\varepsilon}\right]}$ we make

$$
\lim _{R \rightarrow \infty}|\hat{D}(s)| \leq \varepsilon
$$

on the entire arc. Since $\varepsilon>0$ is an arbitrary small positive number, setting $\varepsilon \rightarrow 0$ proves that $\hat{D}(s)->0$ at $|s| \rightarrow \infty$ in the right plane of $\operatorname{Re}(s)>0$.

\section{APPENDIX B}

An explicit calculation may be made to characterize the number of growing modes versus the detuning. The real part of the dispersion relation is given by

$$
\hat{F}^{\prime}\left(\hat{\Delta}-\xi_{i}\right)=0,
$$

where the detuning must be defined in reference to some characteristic energy, and $\xi_{i}$ characterizes some root.

Let us consider how windings appear and disappear topologically. Running from $\xi=-\infty$, the first extremum must be a local maximum given our assumptions. Denote its location by $\xi_{o}$. Now consider

$$
\operatorname{sgn}\left(\mathcal{P} \int \frac{d \hat{F}}{d \hat{P}} \frac{d \hat{P}}{\left(\hat{P}+\xi_{o}-\hat{\Delta}\right)}\right) .
$$

If this sign is positive, the contour crosses in the upper half-plane. If it is negative, it crosses in the lower halfplane. Now consider the next point, a local minimum. This is crossing back over from the left half-plane to the right half-plane. Consider the same question of sign. If its sign has changed then the contour has crossed back over around the origin in the opposite half-plane.

If we make a table, then the first two crossings can be either $(++),(+-),(-+)$, or $(--)$. The $(+-)$ configuration adds one to the winding number, and is the first configuration due to the derivative of the dispersion function at the top of the contour. The next one can be either $(--)$ or $(-+)$. The $(--)$ does not affect the winding number, while the $(-+)$ increases it by one. The next sign in the sequence must always match the previous sign, and the number of these pairs with opposite signs corresponds to the winding number. The total number of windings can be calculated by keeping track of how many times the contour crosses from the one half-plane to the other, and how. Therefore, the number of growing modes and their critical points are intimately related to the sign of the above principal value integral at each crossing. 
As an example, consider the contour in Fig. 7. Following with the discussion above, the first crossing is above the origin, and it crosses back below the origin, so its sign is $(+-)$. It then crosses the vertical axis above the origin again, and then back below the origin, so that is a $(-+)$. Finally, there is a crossing below the origin which then crosses back below the origin, so this is a $(--)$. The table then reads $(+-,-+,--)$. The last crossing does not affect the winding, the first $(+-)$ corresponds to an increase in the winding number by one, and the second one adds another one, as it is looping back again.

If, on the other hand, the sequence were $(+-)$ followed by $(--)$ and another $(--)$, as would be the case if the detuning where changed and therefore vertically shifted the contour, this would correspond to a winding number of one, and therefore one growing mode. This gives a simple way to compute numerically the winding number without resorting to the contour plots, although it is not as intuitive or enjoyable.

[1] J. M. J. Madey, J. Appl. Phys. 42, 1906 (1971).

[2] D. Deacon, L. Alias, J. M. J. Madey, G. Ramian, H. Schwettman, and T. Smith, Phys. Rev. Lett. 38, 892 (1977).

[3] P. Emma, in Proceedings of the 23rd Particle Accelerator Conference, Vancouver, Canada, 2009 (IEEE, Piscataway, NJ, 2009), p. 3119.

[4] M. Altarelli et al., DESY Technical Report, 2006.

[5] S. Goto et al., RIKEN Technical Report, 2010.

[6] S. Reiche, Ph.D. thesis, University of Hamburg, 1999.

[7] W. Fawley, LBNL Technical Report, 1995.
[8] A. M. Kondratenko and E. L. Saldin, Part. Accel. 10, 207 (1980) [http://cdsweb.cern.ch/record/1107977].

[9] R. Bonifacio, C. Pellegrini, and L. Narducci, Opt. Commun. 50, 373 (1984).

[10] E.L. Saldin, E. A. Schneidmiller, and M. V. Yurkov, Nucl. Instrum. Methods Phys. Res., Sect. A 313, 555 (1992).

[11] Y. H. Chin, K.-J. Kim, and M. Xie, Phys. Rev. A 46, 6662 (1992).

[12] E. L. Saldin, E. A. Schneidmiller, and M. V. Yurkov, The Physics of Free Electron Lasers (Springer, BerlinHeidelberg-New York, 2000).

[13] N. Kroll and W. McMullin, Phys. Rev. A 17, 300 (1978).

[14] S.D. Webb and V.N. Litvinenko, in Proceedings of FEL'10 Conference (2010), p. 56 [http://accelconf.web .cern.ch/AccelConf/FEL2010/papers/mopb03.pdf].

[15] A. Vlasov, Sov. Phys. Usp. 10, 721 (1968).

[16] L. D. Landau, J. Phys. USSR 10, 25 (1946).

[17] H. Nyquist, Bell Syst. Tech. J. 11, 126 (1932).

[18] L. A. MacColl, Fundamental Theory of Servomechanisms (Van Nostrand, New York, 1945).

[19] O. Penrose, Phys. Fluids 3, 258 (1960).

[20] P. A. Sturrock, Phys. Rev. 112, 1488 (1958).

[21] R. J. Briggs, Electron-Stream Interaction with Plasmas, MIT Press Classics Series (MIT Press, Cambridge, MA, 1964).

[22] R. C. Davidson, An Introduction to The Physics of NonNeutral Plasmas (Addison-Wesley Publishing Co., Reading, MA, 1990).

[23] G. Wang et al., Asymptotic Behavior of 1D FEL Dispersion Integral at Large $|s|$, C-AD Accelerator Note 428, 2011 [http://www.cadops.bnl.gov/AP/ap_notes/ap_note_ 428.pdf]. 